\title{
Development of Protocols Suitable for Atomic-Scale Imaging of Catalyst Clusters at Catalytic Temperatures
}

\author{
Louis T. Germinario* and Lawrence F. Allard** \\ *Eastman Chemical Company, Kingsport, TN 37662 \\ **Materials Science \& Technology Div., Oak Ridge National Laboratory, Oak Ridge, TN 37831
}

Recent advances in aberration corrected electron microscopy (ACEM) are providing new tools and opportunities for studying chemical processes at gas-solid interfaces at atomic resolutions. In the area of heterogeneous catalysis, STEM-based high-angle annular dark-field imaging (HAADF) has provided the potential for direct observation of single heavy atoms and atom clusters. [1]. This approach has been used to study catalytic properties by directly probing structure development as a result of metal particle-substrate interactions, and used to explore the combined effects of temperature, promoters, and organic ligands on the evolution of catalyst structure [23]. Associated with this new tool is the potential for artifacts, such as electron beam-induced polymerization of organic components [4], and the influence of the electron beam on heavy atom diffusion and nanostructuring [5]. Experiments were conducted in order to develop sample preparation techniques and imaging protocols that are suitable for achieving sub-Ångström resolutions at ambient temperatures. In-situ heating studies were also conduct using Aduro ${ }^{\mathrm{TM}}$ heater devices manufactured using MEMS technology by Protochips Inc. (Raleigh, NC) [6].

Atomic-scale imaging of small molecule-metal cluster interactions in situ under catalytic temperatures was recently initiated [7], using the aberration-corrected JEOL 2200FS STEM/TEM instrument, equipped with a CEOS GmbH corrector on the probe-forming lenses, housed in ORNL's Advanced Microscopy Laboratory. After exploring sample preparation protocols suitable for atom-level imaging at ambient temperatures, experiments were conducted to identify and minimize the inadvertent sources of organic contaminants introduced with the sample during preparation. Sigma-Aldrich, $5 \% \mathrm{Ru}$ on carbon was the source of catalyst materials used in this study. Catalysts were deposited from an alcohol suspension onto holey carbon films supported on either $\mathrm{Cu}$ grids or Protochips Aduro ${ }^{\mathrm{TM}}$ devices. Beam irradiation effects were studied by repeated scanning of suitable areas using the normal high-resolution mode. A partial sequence of images is shown in Fig. 1. Single Ru atoms (arrows) and amorphous atom clusters (rafts) are clearly evident and undergo extensive ordering and crystallization after repeated scanning that is reminiscent of Ostwald ripening. The plot in Fig.1 shows a linear dependence of the mean particle diameter with increasing electron scanning time. A similar linear dependence of crystal growth for short thermal heating times $(<30 \mathrm{~min}$.) was also reported in studies on sintering behavior of Pd nanoparticles [8]. In-situ heating experiments (Fig. 2) conducted in the microscope column using Protochips Aduro ${ }^{\mathrm{TM}}$ devices provided evidence for a temperatureinduced migration, coalescence and ordering at $200^{\circ} \mathrm{C}$, that is independent of electron-beam induced effects.[9]

\section{References}

[1] M. Isaacson, J. Langmore, N. W. Parker, D. Kopf and M. Utlaut, Ultramicroscopy 1 (1976) 359-376.

[2] E.D. Boyes, P. L. Gai, Ultramicroscopy 67 (1997) 219.

[3] R. Sharma, P. A. Crozier, Environmental Transmission Electron Microscopy in Nanotechnology,

Microscopy in Nanotechnology, Kluwer Academic, New York, 2005. 
[4] M. Isaacson, D Kopf, M. Ohtsuki and M Utlaut, Ultramicroscopy 4 (1979) 97-99.

[5] L.T. Germinario, R. Reed, M.D. Cole, S.D. Rose, J.W. Wiggins, M. Beer, S.E.M. 1 (1978) 69-76.

[6] L. F. Allard, W. C. Bigelow, M. Jose-Yacaman, D. P. Nackashi, J. Damiano and S. E. Mick, Micros Res \& Tech 2009, 72(3) in press.

[7] WFO agreement No. NFE-07-01072 between UT-Battelle, LLC and Eastman Chemical Company.

[8] J. J. Chen, E. Ruckenstein, J. Catalysis 69 (1981) 254-273.

[9] Research at the Oak Ridge National Laboratory's High Temperature Materials Lab was sponsored by the U. S. DOE, Office of Energy Efficiency and Renewable Energy, Vehicle Technologies Program.
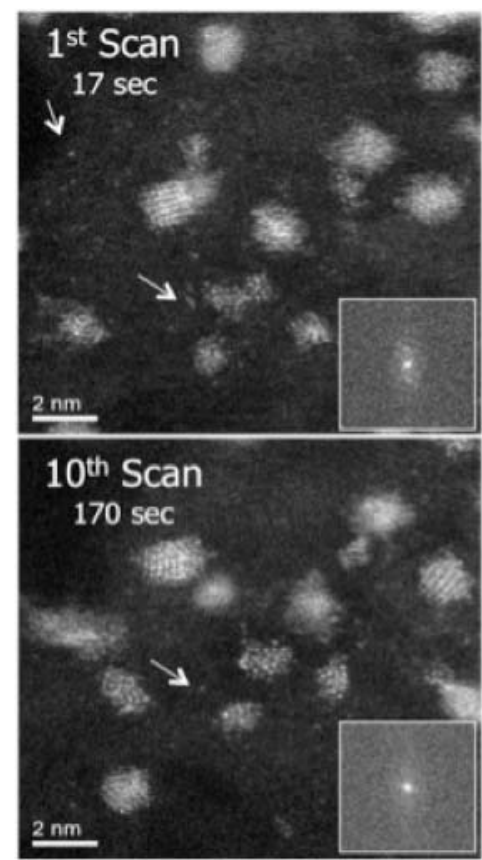
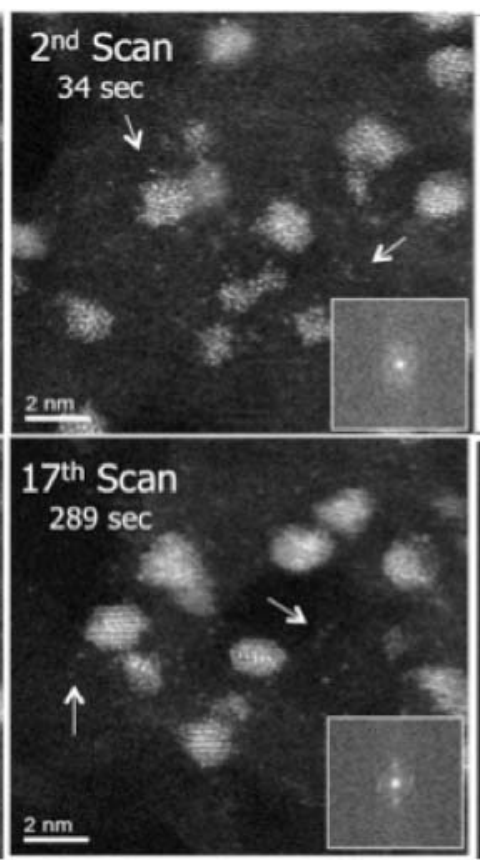
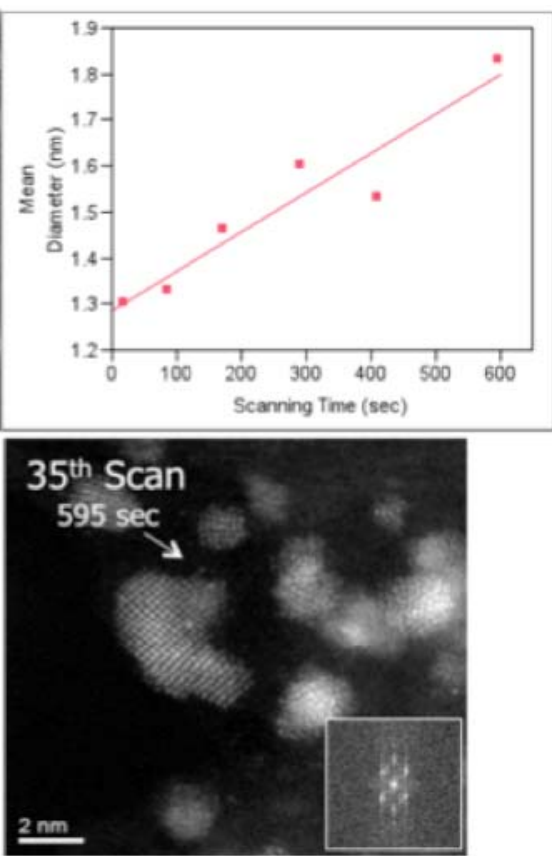

Fig. 1. Electron irradiation effects on diffusion of single atoms (arrows). Amorphous atom clusters (rafts) grow in size and develop crystalline domains after extensive scanning. Plot show mean particle diameters as highly correlated $\left(\mathrm{R}^{2}=0.90\right)$ with electron scanning times.
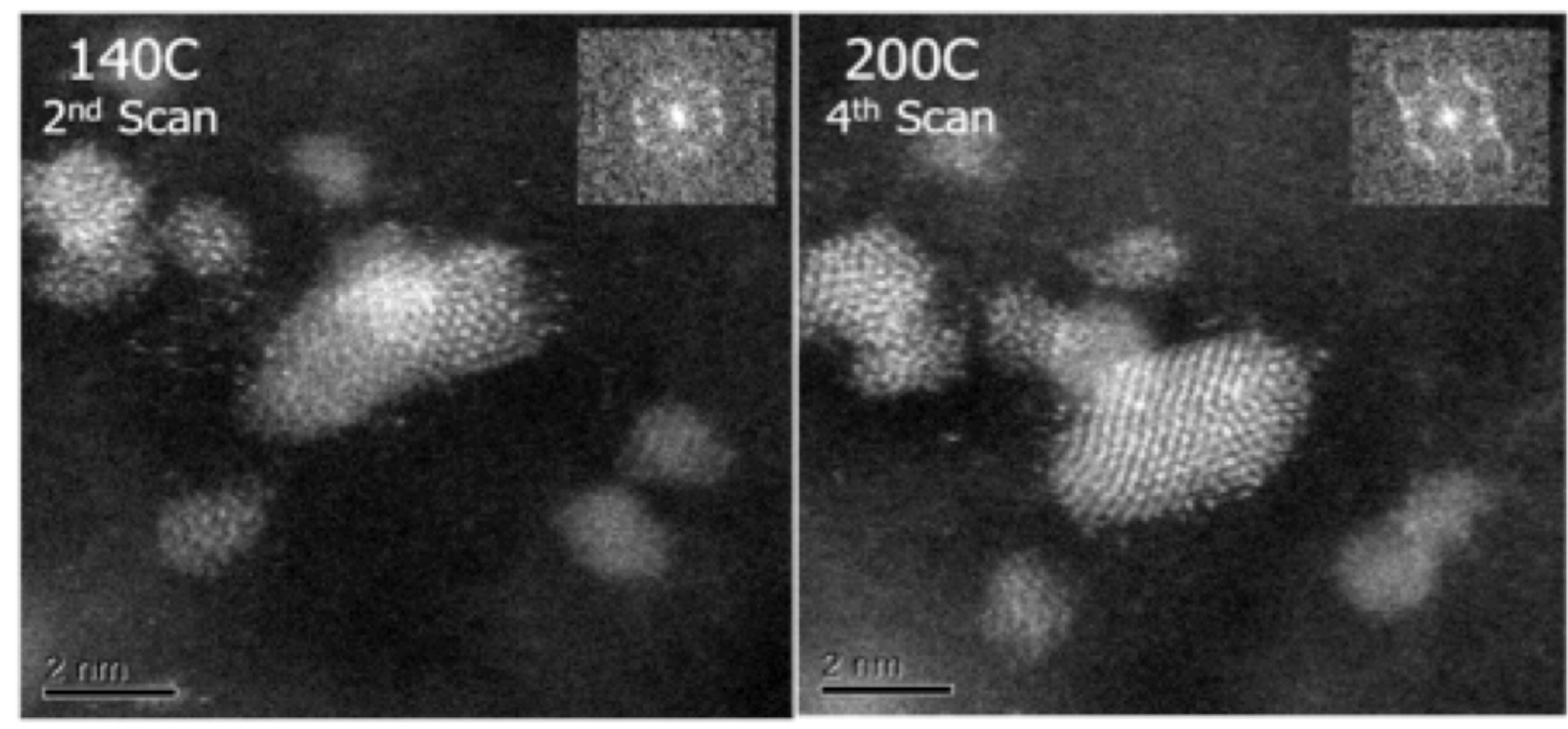

Fig. 2. In-situ heating of $\mathrm{Ru} / \mathrm{C}$ catalyst preparations, in vacuum, provided evidence for a temperature-induced migration, coalescence and ordering at $200^{\circ} \mathrm{C}$, that is independent of electron-beam effects. 